\title{
El proceso de sensibilización como metodología para la aceptación y reconocimiento del ser
}

\section{The sensitizing process as methodology for the acceptance and recognition of the being}

\author{
Andrés Enrique Barrera Gaviria ${ }^{1}$
}

\section{Síntesis de la experiencia}

La sensibilización como estrategia para reconocer y a su vez como metodología, ha direccionado la experiencia del proceso de sensibilización hacia la comprensión hermenéutica desde un enfoque reflexivo; plantea la exegesis y la praxis simbólica para el trabajo social y el reconocimiento del ser. El objetivo clave en el Tecnológico de Antioquia -TdeA- es generar espacios de interacción entre sus estudiantes, docentes y directivos, donde se involucre la familia en el proceso para fomentar el respeto por las diversas personas de la institución. Dado qué en el TdeA se han presentado casos de exclusión; por lo tanto se considera importante comenzar a implementar actividades que incentiven al cambio de visión y de pensamiento de las diferentes personas del plantel, pues, es indispensable para rechazar esa brecha de inequidad y de prejuicios sociales por las etiquetas y la estigmatización ${ }^{2}$. La educación, el aprendizaje y la aprehensión, son conceptos fundamentales para fomentar valores éticos, morales, de convivencia e igualdad y formar un ser sensible a las necesidades del individuo y las propias en una sociedad.

Para alcanzar cambios, se parte de actividades y conceptos clave como: accesibilidad, equidad, participación, calidad y pertinencia, pues se pretende construir un plan de acción y atacar la desigualdad. Por ende, es

Licenciado en Educación con énfasis en Humanidades y Lengua Castellana. Docente de la Escuela Colombiana de Negocios. E-mail: gochys2004@gmail.com

2 Cuando se hace referencia a etiquetas y estigmas recoge todo tipo de características físicas, económicas, sociales o culturales que nos diferencian de los estándares sociales o imaginarios. 
Tdea Andrés Enrique Barrera Gaviria

necesario un cambio estructural del imaginario social y comenzar a fomentar con base en actividades de sensibilización para construir actitudes conscientes que reconozcan al otro para concienciar y no volver a otrora histórica sentada en una visión conservadora e uniformadora.

Las actividades y espacios de sensibilización están orientadas a aprovechar la participación activa de todos y todas, para involucrar a la comunidad institucional en acciones que fomenten el respecto, la diversidad de su población y la igualdad de condiciones para sensibilizarse con relación a la diferencia del ser humano a través de estrategias como el cine foro, talleres y discusiones, que perneen a las personas y puntualizar compromisos para la construcción de la política de inclusión.

Gracias a las actividades de sensibilización y a la metodología de la hermenéutica compresiva, el valor y reconocimiento de la diferencia incentivo al cambio de actitud, a la aceptación del individuo y ha reconocer la propia diferencia como un aspecto fundamental en la variabilidad, la mutabilidad y la evolución del hombre desde un aspecto natural que afianza un sistema de valores, creencias, pensamientos y tendencias plurales.

Palabras clave: Sensibilización, diferencia, diversidad, población, sociedad.

\begin{abstract}
The sensitizing as strategy to recognize and at the same time like methodology, operates toward the comprehension hermenéutica since a reflexive focus. Plant the exegesis and the praxis symbolic for the social work and the recognition of being. Educating, to learn and to apprehend, arises with the need to promote moral, ethical values, of contact, acceptance and equality in a society stigmatizing, segregating androtulante. Therefore it is necessary a structural change of the society and to begin to promote a more conscious attitude of the other and not to return to once historic seated in a conservative and unifying vision.
\end{abstract}

The Tecnológico de Antioquia is to strengthen an attitude that values and recognizes the differences, starting to accept from our individuality, our own differences and recognize ourselves as subjects variables, mutable, and proud of a system of values, beliefs, thoughts and trends plural. The Tecnologico de Antioquia intends since its politics to legitimize the differences of the diversity of its population relating them. Conforming thus the formation in the recognition of being "Humanity" and the acceptance as start to reproduce respectful and inclusive attitudes, you consent and unconscious in its students, educational, family and executive.

Keywords: Sensitization, difference, diversity, population, society. 


\section{Contexto de la experiencia}

La realidad en Colombia con respecto a la inserción laboral y educativa de personas con discapacidad física, necesidades educativas especiales, afrodescendientes, indígenas, homosexuales y demás, ha genera la necesidad de múltiples cambios físicos, políticos y culturales; sin embargo el reconocer de la diversidad poblacional ha estado acompañada de cambios arquitectónicos y físicos en todo tipo de contextos. Generando adecuaciones físicas y comunicacionales $^{3}$ de la sociedad para atender a la pluralidad de la población.

Dentro del ámbito laboral, las entidades privadas y estatales han previsto la necesidad de abrir sus puertas a todo tipo de personas, dado los tratados internacionales ${ }^{4}$ y las diferentes políticas que establecen el reconocimiento de la diferencia de las personas como sujetos socialmente funcionales y productivos $^{5}$. El resultado es que no en todos los casos se reconoce la diferencia o se aceptan los gustos, las actitudes, las necesidades, las apariencias o discapacidades ${ }^{6}$, ya que son visualizadas por la sociedad como limitaciones y la verdad es que son meramente limitaciones mentales y espejismos de creencias infundadas por una sociedad capitalista e uniformadora.

Los estereotipos siguen siendo un fenómeno constante en la actitud de las personas, no sólo del Tecnológico de Antioquia si no también de la comunidad en general, pues es un fenómeno o efecto espejo que se refleja en la Institución, porqué la sociedad macro ${ }^{7}$ forma a los individuos y ellos ingresan a la institución con un pensamiento sedimentado y prejuicioso que fomenta la desigualdad y el señalamiento, rotulando a las personas diferentes de raras o de desviadas sin predeterminar diferencias y gustos.

Desde la construcción de actividades y talleres se incentivo el reconocimiento por el otro, se quiso fomentar o poner en marcha iniciativas dirigidas a promover la igualdad de oportunidades entre todos los sujetos que se instituyen en el proceso de ser conscientes de la existencia del yo y del otro.

Olivier Fressard (2006) en su artículo citando a Castoriadis afirma qué:

“...El imaginario social viene a caracterizar las sociedades humanas como creación ontológica de un modo de ser sui generis, absolutamente irreducible al de otros entes. Designa, también, al mundo singular una y otra vez creado por una sociedad como su mundo propio. El imaginario social es un "magma de significaciones imaginarias sociales" encarnadas en instituciones. Como tal, regula el decir y orienta la acción de los miembros de esa sociedad, en la que determina tanto las maneras de sentir y desear como las maneras de pensar. En definitiva, ese mundo es esencialmente histórico. En efecto, toda sociedad contiene en sí misma una potencia de alteridad. Siempre existe según un doble modo:

Comunicacional en el sentido de la divulgación de comerciales y anuncios con un leguaje respetuoso, que reconoce la diversidad como una riqueza, no vista cómo una desviación o desventaja física o cultural.

4 Consultar los diferentes CONPES para la atención a la diversidad de la población, el índice de inclusión para la educación superior y la convención sobre Naciones Unidas (2006) para los derechos de las personas con discapacidad

5 No sólo se hace referencia a las personas con discapacidad, también a los individuos con necesidades educativas especiales, mujeres, homosexuales, transgénero, trasvesti etc. No quiere decir que la diversidad fuese una discapacidad sino que la sociedad sume la diferencia como algo anómalo que no se reconoce y se estigmatiza.

6 Un ejemplo de ello es la discriminación racial, de género o tendencia sexual, también la discapacidad física o las personas con necesidades educativas especiales.

$7 \quad$ La sociedad macro es el contexto general de las instituciones sociales, el estado, las comunidades y las culturas que se reúnen en un conjunto universal. 
el modo de "lo instituido", estabilización relativa de un conjunto de instituciones, y el modo de "lo instituyente", la dinámica que impulsa su transformación. Por eso resulta conveniente hablar de lo "social-histórico" (p.1)

Con ello se deduce qué: el imaginario social recordando los postulados de Hegel y Castoriadis ${ }^{8}$ puede transformarse, por medio de la formación ${ }^{9}$, la educación ${ }^{10}$ y el proceso de sensibilización como metodología para el reconocimiento del ser implementando actividades que formen en valores de respeto, igualdad y aceptación. Es evidente que el imaginario social, está sujeto a ritos, creencias, tiempo e historia; pero con la educación y procesos que conlleven a la toma de conciencia, los estereotipos, la segregación y la indiferencia; se trasformaran en actitudes positivas que consigan un cambio duradero en la sociedad.

Por ello es importante el proceso de sensibilización como estrategia para originar cambios actitudinales y mentales que rompan las cadenas de la uniformidad y la discriminación, involucrando a los agentes sociales para concienciar y adquirir actitudes que conlleven a la igualdad. La sensibilización por ende es un proceso implementado por el Tecnológico de Antioquia, para suprimir esas actitudes de indiferencia o excluyentes, de los estudiantes, docentes y administrativos, incentivando el proceso de comunicación interactiva por el conducto sensibilizador que introduce a un cambio social y duradero, promoviéndose así con ésta estrategia el aprendizaje significativo, pues, se interioriza al estar sensibilizado situándonos en un foco central, logrando así identificar la problemática que se pretende intervenir y aceptar por medio de la reflexión un mensaje que se trasmita claro y práctico a la hora de utilizar talleres, cine foros, foros de experiencias o múltiples formas que incluyan cualquier medio que se procure orientar.

Por ende, la sensibilización, las relaciones temapúblico y la praxis establecen una diada que permite la conceptualización del tema -en éste caso la aceptación, la diversidad y la igualdad arraigan el proceso subjetivo de reconocimiento del otro- generando una comunicación activa y creativa que promueve la interiorización y la retroalimentación constante de lo trabajado, gestando un cambio de actitud y comportamiento en las personas que participan.

Con lo que se ha dicho, el proceso logra que las personas asuman una toma de conciencia con respecto al fenómeno de la discriminación, así pues, la igualdad de condiciones y oportunidades de las personas vulnerables son vislumbradas desde su origen, causa y efecto para sensibilizar a la sociedad con respecto a las injusticias, corrupción e inequidad. Y comunicar la prontitud de un cambio de postura, pensamiento y costumbre, que nos sitúa en un esquema que es polisémico, que informa y es activo que concibe la oportunidad de regresar y hacer catarsis para entablar una conversación amplia sobre el fenómeno y su intervención por parte de los investigadores.

Al establecer un acto comunicativo y una profundización del tema, se logra tocar a la sociedad. Ésta tomara el control de sus decisiones y por consiguiente de sus vidas, aplicando la estrategia de auto determinación, no imponiendo lo que deben hacer o pensar. Proporcionándoles el poder de reflexión que les permitirá actuar según su condición axiológica y antológica.

Lo instituido, institución e instituyente.

9 Formación en el sentido de incentivar valores de igualdad para el individuo tejiendo sociedad, en el hogar y centros educativos, y que se repercute en las universidades y por lo tanto en la comunidad.

10 Educación vista desde el conocimiento teórico e intelectual con una visión cosmogónica que reconozca a los individuos como pares. Educar desde la academia. 
El proceso de sensibilización como metodología, establece varios contenidos básicos que se direccionan a cuatro conceptos base para su aplicación y conceptualización. El proceso ha estado en ejecución durante seis meses; enfatizando en términos como: igualdad, equidad, diversidad y aceptación; siendo los canales para alcanzar un proceso hermenéutico de comprensión que además es defendido por la organización mundial de derechos humanos, que busca eliminar esa brecha que existe entre parámetros uniformadores y reconocimiento de la diferencia.

El proceso se llevo a cabo con tres estrategias metodológicas para el inicio de la sensibilización como reconocimiento del ser. Primero se realizaron cine foros, donde la orientación conceptual facilito la construcción de unas propuestas centradas en la eliminación de barreras, entendidas como implementación para que la institución suprima barreras físicas, actitudinales, y comunicacionales. Instituyendo el cine foro como proceso que conjuga propuestas del público, que giran en torno a la importancia de comprender que la diversidad de la población somos todos, que se establece en conjunto un sistema de actos concretos locutivos, ilocutivos y perlocutivos ${ }^{11}$ lo que permite reconocernos como sujetos institucionales en equilibrio de poderes entre estudiantes, educadores y directivos, para construir una política que señala la intervención de fenómenos que vulneren nuestros derechos. En concreto brindar una valides a la política internacional de los derechos humanos proporcional a las responsabilidades del estado social de derecho, que garantice el pleno desarrollo de todos y todas, y fomente la diferencia no como un impedimento, sino cómo una consecuencia social que no debe invisibilizarse, más bien constituirse como proceso de aceptación, que permita la sensibilización, reconociendo la desigualdad y trabajar en ello como estrategia para que el estado la adopte e integre otras propuestas.

En segunda instancia los talleres, abordan un contacto más personal, estableciendo así la construcción de una política institucional, con base en la opinión, creencia y propuestas de los estudiantes, docentes y directivos. Se organizaron en varias facetas: una etapa de indagación de conocimientos previos o conducta de entrada, que pretendía indagar sobre el concepto de diversidad, cómo se entiende e interpreta; luego un proceso de sensibilización que lo integra, un video con el tema de diversidad para poder concretarlo y confrontarlo con lo especulado en la conducta de entrada; afianzado luego por un proceso de re-conceptualización desde la teoría, concretando lo teórico desde la academia y paralelamente terminar con unos compromisos que afianzan la promulgación y continuidad desde la individualidad.

Y en la tercera parte se resume con un concierto, que fue realizado por jóvenes con discapacidad, cognitiva, auditiva y visual. Que desde su condición trasmiten un mensaje sencillo; -comprender- el reconocer las diferentes capacidades conllevaría a la aceptación propia y por ende la aceptación del otro.

Pasemos ahora, a afrontar la realidad del Tecnológico de Antioquia con conexión a tres categorías, el género, la discapacidad y la raza, observado desde el contexto institucional conlleva a unos análisis de proceso de información y características en estrategia-sensibilización- como en su significación social, confiriendo aceptación de la diferencia desde el reconocer al individuo y aceptar a los demás.

"Según los actos de habla los tres conceptos se establecen para poder trasmitir la información, además facilita el entendimiento de los espectadores. (Austin 1962) 


\section{Objetivo del proceso de sensibilización como metodología para la aceptación y reconocimiento del ser}

El objetivo de ésta estrategia consiste en aprovechar espacios de participación, como el cine foro, talleres y foros para confluir a la institución en actividades que provoque el respecto, la diversidad de su población y la igualdad de condiciones, para sensibilizar, pero también para construir una política que sea acorde a las necesidades de la variabilidad del contexto y de los entes sociales.

Después de todo la sensibilización busca implementar un cumplimiento viable a los acuerdos internacionales y políticas estatales, enfocadas a buscar la igualdad de condiciones no sólo entre razas, género o discapacidad, sino también entre todos y todas.

La ley 1346 de 2009 hace énfasis en unos puntos concretos sobre los derechos de las personas con discapacidad, pero también busca el reconocimiento de la diferencia, de las disímiles capacidades y de intervenir con integridad reconociendo la vulnerabilidad como uno de los múltiples fenómenos atacar.

En resumen establece: "El propósito de la presente Convención es promover, proteger y asegurar el goce pleno y en condiciones de igualdad de todos los derechos humanos y libertades fundamentales por todas las personas con discapacidad, y promover el respeto de su dignidad inherente.

Las personas con discapacidad incluyen a aquellas que tengan deficiencias físicas, mentales, intelectuales o sensoriales a largo plazo que, al interactuar con diversas barreras, puedan impedir su participación plena y efectiva en la sociedad, en igualdad de condiciones con las demás". Convención sobre los derechos de las personas con discapacidad. (p. 6)

A partir de la interpretación de la ley 1346, que da pie a trabajarse desde cualquier contexto, reconociéndose y sustentándose en los derechos humanos y en la constitución política Colombiana de 1991 se infiere que: el proceso de sensibilización comprende varios pasos; a) formar sujetos sensibles al fenómeno de vulnerabilidad b) reconocer que en algún momento cualquier persona incluyéndose, pudiese estar en una situación de vulnerabilidad y c) fomentar el reconocimiento de la diferencia, no cómo un problema, sino mostrarse conforme a una realidad social, además, de repensar nuestro rol. En consecución se quiere un alcance donde las estrategias que se adquieren en el proceso faciliten la solución pertinentemente de los fenómenos de exclusión.

¿Qué más propone la estrategia? Multiplicar el proceso de sensibilización con otro tipo de actividades que enmarque acciones específicas del rol, que se recapitule la realización de actividades de difusión y promoción de los derechos humanos de todos y todas. Un aspecto clave es que los estudiantes, docentes, administrativos y familias se sientan respaldados por la institución para promover actividades que convoquen a movimientos alternativos de seguimiento al proceso de sensibilización del ser.

Todo esto nos dice que, el proceso debe tener un acompañamiento continuo y un encadenamiento en recursos, pues es fundamental para que no se pierda el sentido del proceso de sensibilización en busca del reconocimiento del ser. Por lo tanto, es fundamental el registro de los resultados, -¿Qué se alcanzo?- y tomar las propuestas para fortalecer las herramientas y medidas institucionalizadas, detectando nuevas necesidades del ámbito y 
facilitar según el rol, alternativas de intervención pertinentes, que se establecen en la creación de la nueva y naciente política institucional, para la igualdad de condiciones.

La sensibilización ofrece: a) contenidos y material del proceso propositivo para la creación del plan de acción, b) propuestas para el diseño de la política institucional de atención a la diversidad de la población y c) el reconocimiento de la diferencia. Todo ello establece compromisos que consiguen la eliminación de barreras físicas, actitudinales y comunicacionales en el Tecnológico de Antioquia; además, multiplica una actitud inclusiva y de igualdad por parte de la creación del plan de acción y la política propuesta por los estudiantes, docentes, administrativos y familias.

La asesoría y el acompañamiento para adquirir estrategias direccionada en atender la diversidad de la población, compete unos compromisos que ayudan al desarrollo y elaboración de informes de seguimiento de los diferentes casos presentados en la institución, para que estos estén al alcance de quienes quieran acceder a él.

\section{Las teorías que la sustentan}

En el ultimo foro de educación para todos según la "DECLARACIÓN EPT" afirma que: "Hace ańos las naciones de la tierra a través de la declaración Universal de los Derechos Humanos afirmaron que "toda persona tiene derecho a la educación”. A pesar de los importantes esfuerzos realizados por los países a lo largo del mundo entero para asegurar el derecho a la educación para todos, persisten las siguientes realidades: 100 millones de niños y de niñas sin acceso a la enseńanza primaria (60\% son niñas). 960 millones de adultos (60\% mujeres) son analfabetos. Analfabetismo funcional grave problema en todos los países incluyendo los industrializados. $30 \%$ de adultos del mundo carece de acceso al conocimiento letrado y a las nuevas habilidades y tecnologías. Más de 100 millones de niños y adultos fracasan en completar los programas de educación básica; otros millones asisten pero no aprenden".

(p. 5-6)

Por eso es necesario tomar de la experiencia y de la política para afianzar procesos sensibilizadores que conlleva al cambio de percepciones, por ende, se facilita el acceso a la educación de todos y todas, y comenzar el proceso de aceptación e igualdad de condiciones.

Aunque el concepto de sensibilización se manifieste desde su constructo teórico como: "Mecanismo por el que la respuesta inmune provocada por un antígeno aparece con mayor intensidad tras una administración inicial" Hernández Hernández. (2003 p. 2). La sensibilización va más allá de su atadura a los términos biológicos, pues, es más una capacidad que se puede ser formada por medio de la reflexión enfocada a un carácter social y humano de lo subjetivo; brindando así la posibilidad de concebir tejido social que dirija a recapacitar sobre, el valor y la importancia del otro como ser. El proceso de sensibilización es arduo y extenso, mas es complejo debido a la escases de recursos para poner en práctica su contenido social, pues requiere capacitación humana la cual puede llevar a cabo la labor pertinentemente, por lo tanto implicaría un cambio, una evolución y una trasformación social, cultural y educacional, mediante percepciones ya sedimentadas desde la formación, -actitudes propias de los prejuicios del ser y estereotipos de los estamentos sociales-.

En las teorías sociales, éste proceso ha llevado a cambios demográficos, políticos, económicos 
y educativos, pues sus planteamientos han concebido un reto difícil para la sociedad y se enmarcaría en la convivencia con otras culturas, dado el proceso de inclusión y la globalización. En éste sentido la sensibilización se trasforma en un mecanismo imprescindible para concienciar, no sólo a cierta población sino también a personas que por efectos de la migración y el desplazamiento tienen la necesidad de convivir en espacios comunales, donde la intolerancia al gusto y la xenofobia es uno de los principales problemas para la convivencia. Por consiguiente el proceso de sensibilización fundamenta la democracia en beneficio de todos y todas.

En el proceso se recalco el término "convivencia" pues las personas concluyeron que es fundamental incluirlo en la educación universitaria, por eso es indispensable retomarlo en todas las áreas no sólo en las humanidades, ya que las interacciones surgen en los diferentes guetos con el fin de radicar la apertura a nuevos puntos de vista que permita una tolerancia y por ende una convivencia armónica. La sensibilización considera la construcción de vías notables para la interacción y el aprendizaje mutuo entre diferentes percepciones o filosofías, al afirmar que, la convivencia entre individuos, grupos y comunidades influencia al proceso comunicativo ajustando actitudes de respeto que faciliten una praxis fundadora de cambios comportamentales, reconciliando actitudes positivas a favor de la diversidad de la población.

Según Vásquez B (2005, p. 2.): “Diversidad significa variedad, desemejanza, diferencia. En otro sentido. Diversidad significa concurso de varias cosas distintas.

En este contexto no podemos explicar los acontecimientos históricos alrededor de las diferencias, que han estereotipado la conceptualización sobre el funcionamiento humano visto como un fenómeno contra natura por tendencias conservadoras y neoliberales merecedor de discriminaciones. En cambio, cuando entendemos que diversidad es el concurso de cosas distintas la reconocemos como una característica de la persona basada en lo que hace o puede hacer.

La diversidad responde al concepto universal de los seres humanos brindando reflexiones donde se comprende que diferimos en muchos aspectos, personales, físicos, filosóficos, políticos y culturales, que refleja el equitativamente sobre las identidades y su valor".

Con todo y eso, el cambio de percepciones es fundamental en éste proceso, pues desde la concepción educativa se liga directamente aspectos cognitivos, emocionales, creencias, formación y elementos sociales. Que descubren un objetivo: determinar los valores, intereses, motivaciones, necesidades y capacidades que la diversidad de la población expresa. En resumen hay que lidiar con toda esa descarga histórica a priori y posteriori de un conjunto de estímulos condicionados por la tradición, la sociedad, la familia, la institución etc. por lo que partimos del proceso para mediar y alcanzar los objetivos planteados en la metodología -sensibilizaciónque ya esta socialmente en funcionamiento del reconocimiento del ser. 


\section{Fase del proceso:}

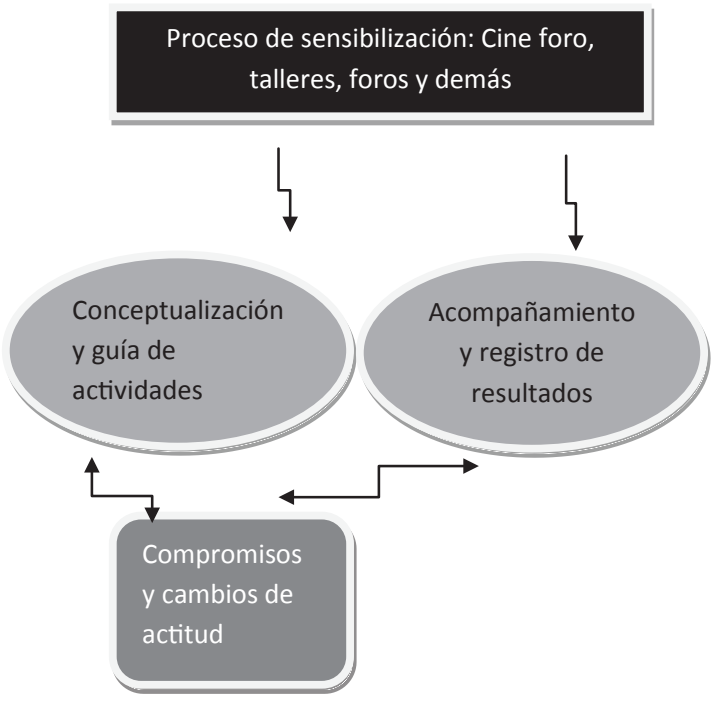

\section{Metodología utilizada en su desarrollo}

El principio base para trabajar la sensibilización como metodología parte de la experiencia obtenida a lo largo del proceso, pues los cine foros, los talleres y el concierto, exteriorizó variables que se identifican como consecuencia de las creencias formativas de los asistentes y establecen así, la visión que se tiene en el Tecnológico de Antioquia sobre "la diversidad" Colmenero Ruiz, (2002, p 72). Por lo tanto, las experiencias previas han arrojando datos que demuestran que la existencia de prejuicios individuales y sociales, establecen creencias falsas sobre diferencias marcadas que hacen creer que el otro diferente a mí, es inferior. Hay fuentes de tipo político que hacen concebir el asistencialismo cómo medida para intervenir y suplir éste fenómeno, fortaleciendo a un más esa brecha y esos dogmas estigmatizantes y etiquetadores frente a ésta realidad. La sensibilización conlleva a generar cierta conciencia por el otro y establece procesos de formación educativa, de aprendizaje y de reconocimiento de las diferentes inteligencias, estilos de aprendizajes y capacidades, alcanzando a identificar la diferencia como una potencialidad -aceptarla desde todas sus dimensiones-.

La realidad del Tecnológico de Antioquia desde el trabajo de sensibilización, instituye cuatro variables que identifican dónde se centra los prejuicios y las actitudes de exclusión:

- Creencias y definiciones equivocas de las diferentes discapacidades.

- Prejuicios sociales desde la formación profesional u rol.

- Información con base en conceptos asistencialistas y comerciales.

- Temor en afrontar la problemática por desconocimiento de estrategias o fundamentación teórica.

La sensibilización como proceso que integra una comunidad a la cual se pretende sensibilizar, es más que un proceso comunal, también es un proceso individual, que hace que el individuo reflexione con respecto al fenómeno que siendo "metafísico" Kant (1783) se convierte en algo colectivo y así la sensibilización actuaría como esa herramienta que incentiva a ése sujeto prejuicioso con conductas y comportamientos colectivos pero educados partiendo de su individualidad y de su interpretación del mundo.

-Si lo que se pretende es generar cierto grado de conciencia por medio de la sensibilización que es una estrategia metodológica-, se modificaría acciones, prácticas y actitudes de exclusión, proporcionando la posibilidad de vivir el fenómeno. Por consiguiente, la teoría configura la interiorización del concepto, con el reflejo de una realidad, en la que se desenvuelve la persona, asumiendo en el proceso de praxis y de exegesis critico-reflexiva, casi autopoiética. 
Para Maturana (1974, pp. 187-196), "la autopoiesis es la propiedad básica de los seres vivos puesto que son sistemas determinados en su estructura, es decir, son sistemas tales que cuando algo externo incide sobre ellos, los efectos dependen de ellos mismos, de su estructura en ése instante, y no de lo externo. Los seres vivos son autónomos, en los que su autonomía se da en su autorreferencia y son sistemas cerrados en su dinámica de constitución como sistemas en continua producción de sí mismos”.

Ciertamente la hermenéutica demarca un enfoque global del significado, pues como metodología del lenguaje, en conjunto con el empirismo, propone una expresión que interprete los fenómenos del mundo Toulmin (1989 p.245-261) “... la ética, valores y la significación de la vida, son susceptibles en una comunicación indirecta o poética”, consolidando a la sensibilización, como mecanismo por el cual el individuo reflexiona y concluirá desde un acto posteriori en el proceso como tal.

\section{Resultados}

A la hora de comenzar a hacer referencia a los resultados, hay que tener en cuenta que hay una serie de aspectos que establecen una continuidad en el paso, además hay la posibilidad de seguir con la propuesta para darle un enfoque social pertinente al proceso.

- La información proporcionada, garantizo e incentivo que se reforzara y alentara, la promoción de actitudes inclusivas, además, de comportamientos positivos que establecen un modelo de repercusión.

- La indagación de saberes previos, aseguro una identificación conceptual del término de diversidad, alcanzando la inferencia de éste y expresando la intertextualidad con otros conceptos afines como: Vulnerabilidad, igualdad de oportunidades, variabilidad contextual, dispersión, género etc. Y así un aprendizaje significativo.

- Se enseñaron y aprendieron prácticas que se requerían para asegurar que los participantes tuvieran la posibilidad de utilizar herramientas didácticas $y$ visuales para un mejor entendimiento del proceso, además de la creación del plan de acción que asumirá el Tecnológico de Antioquia.

- Se proporciono espacios de participación, donde los grupos manifestaron explícitamente sus actitudes, inquietudes y propuestas para un plan de acción institucional, pensado en el otro y en mí, como perteneciente al Tecnológico -actitud inclusiva-

- Se afianzó términos utilizados en el proceso de atención a la diversidad e inclusión, partiendo de la teoría, por eso, se obtuvo la interiorización por medio de la discusión y se consolido así, el aprendizaje significativo.

- Se incentivo a la población a que se comprometiera en pro de un trabajo conjunto para fortalecer y trasmitir la información: respetar y trabajar por la igualdad de condiciones de la diversidad de la población universitaria del Tecnológico de Antioquia nos ayuda a todos.

- Se utilizó diferentes recursos para poder comunicar el proceso, técnica y propósito del Tecnológico de Antioquia para atender a la diversidad de la población y se llego a la mayoría de las personas.

12 El impacto y los resultados se sustentan en los protocolos elaborados en las actividades, el producto de los talleres, en las observaciones del concierto realizado por azul ilusión y en la sistematización de las encuestas del cine foro. 
- Con los acuerdos y compromisos, se produjo una reflexión en las actitudes y se procuró que las percepciones antes y después, puedan trascender del ámbito institucional, al ámbito de la comunidad, la sociedad y la familia.

- Como reflexión se entendió que la pluralidad cultural, de género, física y económica existe, y no hay que pretender olvidar que a lo largo de la historia se ha querido ocultar, pero es inminente su surgimiento, por eso hay que comenzar a trabajar en la atención a la diversidad de la población, reconociendo nuestros militantes, además de nuestras capacidades y así reconocer la diferencia no como una desventaja sino, como esa ventaja de habilidades, esa ventaja cultural de diversidad existente en nuestra sociedad $^{12}$.

\section{Proyecciones de la experiencia}

En el proceso de sensibilización, la formación de los estudiantes y demás entes institucionales asegura, la independencia de pareceres, inferencias y representaciones que establecen características propias del individuo.

El llevar a un plano hermenéutico y simbólico las funciones individuales, establece que en un futuro, el proceso de sensibilización suprima la uniformidad y se direccione a la "equiparación de oportunidades" ONU (1993) para la diversidad de la población en el Tecnológico de Antioquia. Apoyando así el concepto de igualdad como condición para la inclusión y atención.

Además se asegura en el estado social de derecho Colombiano, pues una educación con calidad, debe ser uno de los principales propósitos del Gobierno. La educación en valores éticos y morales proyecta, la formación de sujetos participativos, donde la educación pueda darse en contextos escolares integrados y con igualdad de condiciones.

Se piensa igualmente a futuro con la continuidad de éste proceso conectar la inclusión y la atención a todo tipo de población con programas amplios con un enfoque comunitario para todo tipo de personas, lo que certifica la utilización de los recursos en la comunidad y la permanencia de la diferente población en los programas educativos y formativos.

De alguna manera, el enfoque sensibilizador refleja a largo plazo cambios no sólo en la comunidad universitaria, sino también en actitudes fuera de la institución -factores externos- cómo: la familia, la comunidad, el sector privado, estatal etc. Logrando la reflexión con respecto al ser humano.

Hacer protagonista de un proceso no mercantilista, devuelve el reconocimiento de "él-yo" como ser, observado como persona, con distintas dimensiones de aprendizaje, de competencias y de capacidades.

Si se vuelve atrás, todo esto significa que no se trata sólo de un cambio político y de legislación, implica también orientar la educación a un campo filosófico, metafísico, axiológico y ontológico, determinar por medio del socialismo los principios de igualdad y formar parte de un proceso sistémico, que promueva cambios desde sus fundamentos educativos.

Movilizar a la comunidad estudiantil, docente, familiar y administrativa a construir beneplácitos que analicen situaciones de segregación, identificando las barreras y las oportunidades 
que permita alcanzar beneficios no sólo para la comunidad discapacitada, racial o vulnerable, sino también éxitos para todos y todas.

En un futuro la realización de las actividades debe ejecutarse de acuerdo al beneficio de todos y todas, integrando -como ya ha dicho- una pluralidad de sujetos, que conlleve al aprendizaje mutuo y estimulante -"una labor de equipo"-.

\section{Referencias bibliográficas}

Colmenero Ruiz, Ma Jesús. (2009). Influencia y repercusión de la experiencia como docente en la atención a la diversidad. Revista educación inclusiva vol. 2. No 3. (pp. 71-82).

Colombia. Congreso de la republica. Ley 1346 de. (2009). Por medio de la cual se aprueba la "convención sobre Naciones Unidas (2006) los derechos de las personas con discapacidad", adoptada por la asamblea general de las republica. Disponible en: http://www. colombiaincluyente.org/colombia_incluyente/ userfiles/Ley\%201346\%20de\%202009563.pdf

Hernández Hernández. Fidel. "la sensibilización cultural como estrategia de intervención social". Director de la Escuela de Mediadores Sociales para la Inmigración. Disponible en: http:// www.giemic.uclm.es/index.php?option=com docman $\&$ task $=$ doc_view\&gid $=1008 \&$ Itemid $=60$

Kant, Immanuel (1783). Prolegómenos a toda metafisica futura que pueda presentarse como ciencia. Disponible en varias ediciones.

Maturana, Humberto R. (1976). El árbol del conocimiento. Santiago de Chile: Editorial Universitaria. Primera edición.* Maturana, Humberto. R. (2002). Transformación en la convivencia. Santiago de Chile: Dolmen Ediciones.

Naciones Unidas (2006). Convención sobre los Derechos de las Personas con Discapacidad. “adoptada por la Naciones Unidas. Asamblea General

Olivier Fressard. El imaginario social o la potencia de inventar de los pueblos. Revista trasversales número 2, primavera 2006. Una primera versión de este artículo, en su original francés, fue publicada en la revista Sciences de l'homme \& Sociétés, no 50, septiembre 2005. Disponible En: http://www.fundanin.org/ fressard.htm

Opertti. Renato. (2011). Congreso internacional de educación para todos, los retos de educar en la diversidad. Inclusión docente y docentes inclusivos: ejes para fortalecer una estrategia de cambios. Disponible en: http://www.oei.es/pdf2/ Simposio_Pamplona-09_presentacion.pdf Robalino Campos. Magaly. (2011). "Informe seguimiento de la educación para todos". Educación para todos avances y temas pendiente. Disponible en:

http://www.saldarriagaconcha.org/Library/

News/Files/MAGALY\%20ROBALINO,\%20

EDUCACI\%C3\%93N\%20PARA\%20

TODOS,\%20AVANCES\%20Y\%20T343.PDF

Salas Rodríguez. Claudia Gabriela. (2008). Guía metodológica para la sensibilización en género: Una herramienta didáctica para la capacitación en la administración pública. "La sensibilización en género”. Volumen 1. (pp. 5-33).

Vásquez B, Armando J. (2005). Aprender a Convivir con las Diferencias, La Importancia del Enfoque Inclusivo en las Escuelas. Resumen de la charla presentada por el Asesor Regional de RHB de OPS en el marco del V Congreso Mundial de Educación Inclusiva y I Simposio Internacional de Educación Especial. Venezuela. Disponible en:

http://www.paho.org/Spanish/DD/PIN/ Convivir_con_diferencias.pdf 